\title{
Information integration and compounding of discriminative stimuli in pigeons
}

\author{
PATRICK GRIFFIN, WILLIAM R, HUGHES, and JAMES J. COLEMAN \\ Box 6234, University of Alabama, University, Alabama $\$ 5486$
}

\begin{abstract}
Keypecks were reinforced with food in three pigeons according to a multiple schedule with variable interval schedules of reinforcement associated with the components. If the key was green (G) or white with three horizontal black lines $(\mathrm{H})$, variable interval $30 \mathrm{sec}$ was in effect. A red key (R) or three black vertical lines on a white surround (V) indicated that variable interval 60 sec was in effect. Following this training, a single test session was conducted in which the reinforcer was not available and in which the single stimuli and the compounds HG, HR, VG, and VR were presented. Response rates to the compounds were generally less than to the elements alone. This unexpected result was apparently due to degrading of the stimuli during superimposition. However, the compound rates aligned well with a linear model that assumes no interaction between orientation and color stimuli. This initial agreement with Anderson's information integration approach suggests further application of the model to stimulus compounding in infrahuman animals.
\end{abstract}

Weiss (1972) reviewed current research dealing with compounding of discriminative stimuli in infrahuman subjects. The typical experimental paradigm involved establishment of control over responding by distinct stimuli, usually from different modalities, followed by a test in which the stimuli were combined. Generally, these experiments have attempted to determine the ordinal relation between response rates to the compounds and response rates to the individual elements. Thus, the major interest was whether response rate to the compound was less than the rate to either element (suppressive summation), greater than either element rate (additive summation), or bounded by the element rates (averaging). Since, with a compound presentation, the subject may respond on the basis of a combination of cues, however, the task appears amenable to analysis in terms of information integration theory (cf. Anderson, Note 1). Such analysis would allow a more precise description than the above ordinal classifications by providing a basis for interval measurement.

The information integration model has been applied with human subjects and relatively concrete stimuli, such as judgment of subjective weight of objects (e.g.. Anderson, 1972), as well as with more abstract stimuli, such as the likability of a hypothetical person described by sets of personality traits (e.g.. Anderson, 1962). In a number of these situations, the data have been adequately described by a linear model of the form $R=w_{1} s_{1}+w_{2} s_{2}+\ldots$ $w_{n} s_{n}$ (additive model), where $w$ represents the weight

Reprints may be obtained from the first author. Department of Psychology, Jackson State University, Jackson. Mississippi 39217. We thank Dr. K. L. Norman for suggestions regarding this experiment and preparation of the manuscript. of the stimulus, $s$ the subjective scale value, and $R$ the observed response. By assuming that $w_{1}+w_{2}+\ldots$ $\mathrm{w}_{\mathrm{n}}=1.0$, the model converts to an averaging model (ct. Anderson, 1972).

Both the additive and averaging models are consistent with a noninteractive relation between $s_{1}$ and $s_{2}$ values. That is, if values of $s_{1}$ and $s_{2}$ are combined factorially, the result should plot as two parallel lines, i.e., a nonsignificant Row by Column interaction. Further, since such linear integration models assume that all row stimuli are equally weighted and all column stimuli are equally weighted, the means of the rows and columns may be considered linear functions of their respective subjective scale values. Thus, marginal means constitute an interval scale of the row and column stimuli (functional measurement). Examples of these characteristics of the linear model are provided by Butzin and Anderson (1973).

This approach appears to transfer directly to the stimulus compounding experiments with animals. In a situation in which additive summation is obtained (Weiss, 1971, Experiment 2), for example, the critical issue is whether a stimulus equally increments response rate regardless of the other stimulus with which it is combined. Such an outcome would indicate a noninteractive relation consistent with the information integration model.

Because previously reported compounding experiments have attended only to ordinal relations. however, the results do not lend themselves to such an analysis. Therefore, to exemplify application of the model, the present experiment combined factorially the discriminative stimuli for two schedules of reinforcement in pigeons. 


\section{METHOD}

\section{Subjects}

Three, adult. male Silver King pigeons were maintained at $80 \%$ $( \pm 2 \%)$ of free-feeding body weight. The subjects had histories of discrimination training under various multiple schedules of reinforcement.

\section{Apparatus}

The experimental chamber was $30.0 \mathrm{~cm}$ wide $\mathrm{x} 34.0 \mathrm{~cm}$ deep $\mathrm{x}$ $28.0 \mathrm{~cm}$ high (i.d.). One $2.0-\mathrm{cm}$ clear pigeon key was centered $22.5 \mathrm{~cm}$ above the chamber floor. A $5.5 \times 4.5 \mathrm{~cm}$ aperture, $10.5 \mathrm{~cm}$ above the floor and directly below the key, allowed access to the grain reinforcer.

Stimuli were projected onto the key by an Industrial Electronic Engineers stimulus projector. The stimuli were three vertical black lines on a white surround $(V)$. three horizontal black lines on a white surround $(\mathrm{H})$, a pale red produced by presenting white and red together $(R)$. and a pale green produced by white and green $(G)$. These stimuli were selected from BRS. LVE projector plate No. 111-90-715.

The chamber was equipped with an exhaust fan for noise masking and ventilation and was acoustically isolated from electromechanica! programming and recording equipment.

\section{Procedure}

Training. Since the subjects were experienced with schedules requiring a keypeck response. no shaping was required and the training schedule was imposed immediately. Each subject underwent two 24-min sessions daily. The sessions occurred consecutively and were separated by approximately $10 \mathrm{~min}$. Only two stimuli were used during a particular session, but the stimuli could be any pair of the four possibilities. Three random series of the four stimuli. taken two at a time. generated a total of 18 combinations with each pair appearing three times. Thus. 9 days could occur before the total sequence was repeated. During these sessions, the stimuli occurred separately as components of a two-component multiple schedule (cf. Ferster \& Skinner, 1957).

During all sessions, component duration was $30.0 \mathrm{sec}$ and 41 components occurred per session. The components were separated by a 5.0-sec time-out during which the key was dark and pecks had no scheduled consequences. The components occurred in a semirandom order with the constraint that no more than three presentations of the same stimulus could occur consecutively. Data from the first component of each session were not recorded.

A VI (variable-interval) $30-\mathrm{sec}$ schedule assigned reinforcers during components associated with $G$ and $H$ and a VI $60-\mathrm{sec}$ schedule was operative during $R$ and $V$. That is. on the average of either once every $30 \mathrm{sec}$ (VI $30-\mathrm{sec}$ ) or once every $60 \mathrm{sec}$ (VI 60-sec). a keypeck response produced the reinforcer $15.0-\mathrm{sec}$ access to grain). The intervals comprising these schedules were obtained with the Catania and Reynolds (1968. p. 381) constant probability progression.

Test. Following 36 days ( 72 sessions) of training. a single test session was conducted. During the test, the single stimuli and the combinations RH, RV. GH, and $G V$ were presented, making a total of eight possible stimuli. These stinuli were presented according to an 8 by 8 Latin square with eight randomized blocks of the eight stimuli. With the exception of nonavailability of the reinforcer, the added compound stimuli and the additional session time, the test session was identical to the training sessions.

Certain characteristics of the stimuli should be noted. The $R$ and $G$ stimuli were combined with white during training. This procedure more closely equated the training and test colors. since the white background of the line stimuli was necessarily combined with the colors during the test. On the other hand. during the test. the colors "filled in" the previously black line stimuli. Thus. during the test. the RV compound. for example. appeared as three red verrical lines on a pale red surround.

\section{RESULTS}

Table 1 presents the training and test results for each subject. The training data indicate that the stimuli associated with VI 30-sec ( $G$ and $H$ ) controlled higher response rates than the VI 60 -sec stimuli ( $R$ and $V$ ). Reinforcement frequency was not the only variable determining response rate, however, since there were differences between $G$ and $H$ response rates and between $R$ and $V$ response rates. The rate for $R$ was higher than $V$ in all subjects, and, for two subjects ( $\mathrm{P} 1$ and $\mathrm{P} 3$ ), the rate for $\mathrm{G}$ was above that for $\mathrm{H}$.

Response rates to single stimuli were lower during the test than during training. However, with the exception of $\mathrm{P} 1$, the ordinal relations between stimuli during training were maintained in the test situation.

The final columns in Table 1 indicate that, relative to the test rates for single stimuli, suppressive summation occurred in eight of the 12 compound tests. Further, in those cases where averaging occurred, the rate was only slightly above the lower element rate. No cases of additive summation were observed.

Even though the compounding operation, generally, suppressed responding, the response rates to the compounds approximated parallel functions. This outcome is indicated in Figure 1. For Subjects $P 1$ and $P 2$. parallelism is apparent. The functions for Subject $P 3$ are more divergent but still appear reasonably parallel. Since parallelism indicates a nonsignificant interaction, analysis of variance

Table 1

Responses Per Minute to Stimuli During Training and Testing

\begin{tabular}{|c|c|c|c|c|c|c|c|c|c|c|c|c|}
\hline \multirow[b]{3}{*}{ Subject } & \multirow{2}{*}{\multicolumn{4}{|c|}{$\begin{array}{c}\text { Training } \\
\text { Elements }\end{array}$}} & \multicolumn{8}{|c|}{ Test } \\
\hline & & & & & \multicolumn{4}{|c|}{ Elements } & \multicolumn{4}{|c|}{ Compounds } \\
\hline & $\mathbf{R}$ & $\mathrm{V}$ & G & $\mathrm{H}$ & $\mathbf{R}$ & $\mathrm{V}$ & $\mathrm{G}$ & $\mathbf{H}$ & RV & $\mathrm{RH}$ & GV & $\mathrm{GH}$ \\
\hline P1 & 78.7 & 72.0 & 104.0 & 85.3 & 43.5 & 42.8 & 72.5 & 62.3 & $21.8(\mathrm{~s})$ & $30.0(\mathrm{~s})$ & $49.3(\mathrm{a})$ & $58.8(\mathrm{~s})$ \\
\hline $\mathrm{P} 2$ & 46.2 & 43.1 & 68.0 & 74.0 & 28.2 & 31.5 & 71.8 & 65.8 & $14.8(\mathrm{~s})$ & $27.5(\mathrm{~s})$ & 35.5 (a) & $53.0(\mathrm{~s})$ \\
\hline P3 & 89.8 & 64.4 & 120.0 & 116.3 & 68.8 & 58.0 & 120.5 & 98.5 & $52.0(\mathrm{~s})$ & 70.8 (a) & $62.0(\mathrm{a})$ & $92.3(\mathrm{~s})$ \\
\hline
\end{tabular}

Note-The rates to the compounds are classified as suppressive summation (s) or averaging (a) relative to the element rates during testing. 


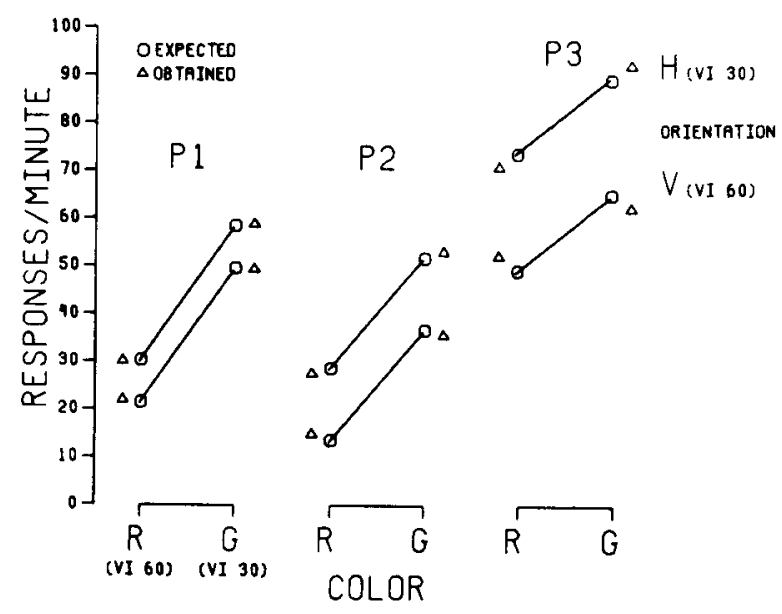

Figure 1. Obtained response rates to compound test stimuli and expected rates based on the linear model for Subjects P1, P2, and $P 3$. The obtained rates have been laterally displaced for clarity.

provides a further and more rigorous test. A two-way randomized blocks ANOVA, i.e., Color by Orientation over the eight test blocks, was conducted for each subject. In all cases, the Color by Orientation interaction was nonsignificant [Subject $\mathrm{P} 1, \mathrm{~F}(1,7)=$ $.04, \mathrm{p}>.2$; Subject $\mathrm{P} 2 . \mathrm{F}(1,7)=.12, \mathrm{p}>.2$; Subject P3, $F(1,7)=2.5, \mathrm{p}>.1]$.

The degree of parallelism can also be illustrated by using the obtained row and column means to "predict" the cell entries expected given no interaction. It can be shown that the expected value for a particular cell equals the respective row mean plus the column mean minus the grand mean (cf. Butzin \& Anderson, 1973). Figure 1 illustrates the discrepancy between the obtained and expected values. In only Subject P3 were the deviations appreciable.

\section{DISCUSSION}

The result that response rates to single stimuli were less during the test than during training is not too surprising since extinction was in effect and no reinforced warm-up trials were given. Somewhat more unexpected was the preponderance of suppressive summation or slight averaging effects between test elements and compounds. Both Weiss $(1964,1971)$. with light and tone compounds, and Wolf (1963), with light intensity compounds, reported additive summation when the discriminative stimuli for separate VI schedules were combined. However, the subjects in these experiments were rats. Further, Miller (1971) reported reduced additive summation for intramodal compounds, as in the present case and in Wolf (1963) relative to intermodal compounds.
Perhaps the most critical factor was alteration of the stimulus elements due to superimposition during compound tests. Although superimposing the stimuli upon the response operandum was an attempt to insure that the birds attended to both elements, the procedure degraded the stimuli to some extent. This degradation may have produced the overall reduction in response rate (see Weiss, 1972, p. 193).

These issues notwithstanding, the functions for responding to the compounds were reasonably described by parallel lines, i.e., the addition of an element from one dimension produced the same increment in response rate regardless of the value of the other dimension. This concordance with integration theory was further illustrated by using the obtained row and column means and the linear model to generate cell entries under the assumption of no interaction. The agreement was quite close for two subjects.

The information integration model thus provided a reasonable description of how the elements of the stimulus compound were combined by the subjects to determine rate of responding. A number of factors, however, temper the generality of these data. Presenting only two values on each dimension provides a rather limited test of parallelism. In addition, current concern regarding the peculiarities of the keypeck response in pigeons (Moore, 1973) raises the issue of extension across species and operants. Finally, whether similar data would obtain if the dependent variable were a choice measure (e.g., Peterson \& Premack, 1971) rather than response rate to a single operandum is an open question. These issues appear readily tractable to investigation.

\section{REFERENCE NO'TE}

1. Anderson, N. H. Algebraic models in perception. Technical Report No. 24, Center for Human Information Processing, University of California, San Diego, 1972.

\section{REFERENCES}

ANDERSON, N. H. Application of an additive model to impression formation. Science, 1962, 138, 817-818.

ANDERson, N. H. Cross-task validation of functional measurement. Perception \& Psychophysics, 1972, 12, 389-395.

Butzin, C. A.. \& Anderson, N. H. Functional measurement of children's judgments. Child Development, 1973, 44, 529.537.

Catania, A. C., \& Reynolos, G. S. A quantitative analysis of the responding maintained by interval schedules of reinforcement. Journal of the Experimental Analysis of Behavior. 1968. 11 , 327-383.

Ferster, C. B., \& Skinner, B. F. Schedules of reinforcement. New York: Appleton-Century-Crofts, 1957.

Miller, L. Compounding of discriminative stimuli from the same and different sensory modalities. Journal of the Experimental Analysis of Behavior. 1971. 16, 337-342. 
Moore. B. R. The role of directed Pavlovian reactions in simple instrumental learning in the pigeon. In R. A. Hinde and J. Stevenson-Hinde (Eds.). Constraints on leaming: Limitations and predispositions. New York: Academic Press. $19^{-} 3$.

Peterson. J.. \& Premack. D. A method of mapping stimulus distance into reinforcement value. Learning and Motivation. 1971. 2. 40-48.

Weiss. S. J. Summation of response strengths instrumentally conditioned to stimuli in different sensory modalities. Journal of Experimental Psychology. 1964, 68. 151-155.

WEIsS. S. J. Discrimination training and stimulus compounding:
Consideration of non-reinforcement and response differentiation consequences of $\mathrm{S} \triangle$. Journal of the Experimental Analsis of Behavior, 1971, 15, 387.402.

WEISS, S. J. Stimulus compounding in free-operant and classical conditioning: A review and analysis. Psychological Bulletin, 1972, 78. 189-208.

Wol.F. M. M. Some effects of combined $S D_{S}$. Journal of the Experimental Analysis of Behavior. 1963, 6. 343-347.

(Received for publication September 9. 1974; revision received February $7,1975$. 